\title{
Resúmenes de trabajos de investigación presentados en la jornada científica por los 41 años de la Facultad de Estomatología, UPCH
}

Proyecto: GERES-UPCH: "Generating and Developing Research Proyects at the Faculty of Stomatology - UPCH"

\author{
Coordinador de Proyecto GERES - UPCH: Lic. Javier Mendoza Paitán \\ Coordinadora de Proyectos - Química: ～Ing. Gilma Fernández Zúñiga
}

Los resúmenes que se presentan a continuación corresponden a proyectos de investigación planteados dentro del Programa denominado "Desarrollo de Proyectos de Investigación a través de Grupos de Trabajo con Estudiantes del Primer Año de la Facultad de Estomatología Roberto Beltrán", contando con la participación de los ingresantes 2010 - I y de Docentes de la Facultad de Ciencias y Filosofía en calidad de Asesores de Investigación. Este programa se ha desarrollado dentro del marco del Proyecto GERES UPCH, "Generating and Developing Research Proyects at the Faculty of Stomatology - UPCH", dirigido a generar los medios y espacios necesarios para la formación, participación y realización de las diferentes fases del proceso de investigación de proyectos orientados al desarrollo tecnológico y científico en la Facultad de Estomatología Roberto Beltrán.

\section{Efecto de la temperatura en la actividad de catalasa para 5 variedades de papas nativas. \\ Mendoza, J.* Nieto, S. Najarro, M. Mellado, J. Hidalgo, J. \\ Vidal, M.* \\ *Asesores de Investigación, Proyecto GERES-UPCH}

La catalasa en una enzima importante por su función antioxidante ya que bloquea el efecto de los radicales libres causantes del envejecimiento celular. Al no poseer catalasa, el organismo acumula peróxido de hidrógeno, como ocurre en la acatalasemia (Takahara, 1952), la cual produce inflamación y sangrado de las encías. De ahí la importancia del consumo y estudio de alimentos ricos en antioxidantes como la papa (Solanum tuberosum). El objetivo del presente trabajo fue evaluar el efecto de la temperatura de congelamiento en la actividad de catalasa en 5 variedades de papa: "amarilla", "rosada", "peruanita", "perricholi" y "negra". Se consideraron los casos a temperatura ambiente $\left(15^{\circ} \mathrm{C}\right)$ y temperatura de congelamiento de $-20^{\circ} \mathrm{C}$. Se midió el tiempo de reacción y la altura de la espuma alcanzada en los tubos de ensayo como parámetros para evaluar el grado de actividad de la catalasa. A temperatura ambiente el menor tiempo en lograr las mayores alturas se obtuvo con las papas "negra" y "amarilla" (35 s y 38 s respectivamente), siendo la "amarilla" la de mayor altura en la reacción. A - $20^{\circ} \mathrm{C}$ el menor tiempo en lograr las mayores alturas lo obtuvieron las papas "rosada" y "amarilla" (53 s y 65 s. respectivamente), siendo la "rosada" la de mayor altura en la reacción. Es posible concluir que la papa "amarilla" presenta una mayor actividad de catalasa", la cual es afectada por la temperatura de congelamiento de $-20^{\circ} \mathrm{C}$, por lo que se requieren estudios posteriores para su cuantificación.
Palabras clave: papas nativas, catalasa, acatalasemia.

Simulación en GEANT4 para la detección de caries oclusales usando el láser ER:YAG.

Altamirano, A.* Shon, F. Vinces, K. Vilchez, K. Rodriguez, A. Zapata, G.

*Asesor de Investigación, Proyecto GERES-UPCH

El LASER (Light Amplification by Stimulated Emission of Radiation) es un dispositivo muy usado en la actualidad en las diferentes áreas de la odontología, para el blanqueamiento de piezas dentarias, endodoncia y cirugía debido a que es una técnica que en muchos casos no necesita anestesia y el tiempo de operación y cicatrización es muy corta. El objetivo del presente trabajo fue desarrollar la simulación de la irradiación de una pieza dentaria usando el láser Er:YAG (láser de Erbio) orientada al estudio de la detección de caries oclusales. Para la simulación se usó el incisivo inferior extraído de un cráneo de niño, al cual se le tomaron las medidas espaciales necesarias. Se trabajó con este laser por ser el más reciente de aplicación en las diferentes especialidades de odontología. La implementación se realizó usando el entorno de simulación GEANT4, formado por una serie de librerías en C++ y programación orientada a objetos, usado frecuentemente en física de altas energías, medicina y biología e instalado en la distribución Linux OpenSuse 11.3. Se obtuvieron las librerías de simulación de los dientes y del láser a fin de realizar posteriores pruebas.

Palabras clave: láser, GEANT4, simulación. 
Saturación del esmalte dental respecto a la adsorción de ácido carmínico y tartrazina.

Guzmán, E.* Gómez, C. García, J. Gonzales, A. Guerra, K. Calvay, $\mathrm{K}$.

*Asesor de Investigación, Proyecto GERES-UPCH

El objetivo del presente trabajo fue evaluar la saturación del esmalte dental respecto a la adsorción de Acido Carmínico y Tartrazina. Para determinar la cantidad de colorantes adsorbido por el diente se prepararon soluciones estándares de cada colorante y una vez añadido el diente se midieron las concentraciones de las soluciones en el espectrofotómetro Ultravioleta - Visible, obteniéndose por diferencia de concentraciones la cantidad de colorante adsorbido por el diente. Se observó una mayor adsorción del colorante ácido carmínico con respecto a la tartrazina. La estructura del acido carmínico presenta un mayor número de oxígenos capaces de coordinar al calcio del esmalte dental, lo cual le permite enlazarse a este con una mayor fortaleza que la tartrazina que posee nitrógenos poco afines por el calcio. Se determinó el tiempo de vida media para cada colorante, de modo que para el ácido carmínico fue de 3241 min y para la tartrazina de 1726 min. Es importante señalar que la coloración producida en el diente por dicha cantidad resulta imperceptible.

Palabras clave: Adsorción, colorante, espectrofotómetro.

Aplicación de métodos de ajuste en busca de simetría facial. Sisniegas, G.* Verano, M. Rivera, A. Ruiz, A. Vera, L. Samaniego, A.

*Asesor de Investigación, Proyecto GERES-UPCH

Se ha desarrollado un método automático que ayude en el reconocimiento de estados faciales temporales y/o permanentes de utilidad en el análisis de rostros, de sus patologías y las diferentes configuraciones faciales que un rostro suele adquirir durante la comunicación social o determinado estado anímico. Debido a que el estudio estético de un rostro tiende a ser arbitrario y complicado, se requiere de una referencia objetiva basada en algún criterio matemático, por lo que se dispuso utilizar un método de ajuste lineal no tradicional mediante el cual se estipula la resolución sin ambigüedad de la línea recta ajustada a una determinada población de puntos dispersos en un plano. Este método fue desarrollado estableciendo una analogía entre la distribución de puntos en un plano en relación a su centro con la aplicación de fuerzas coplanares. Las ecuaciones resultantes (dos líneas o ejes de equilibrio) permiten comparar su posición y su inclinación respecto a las referenciales (típicamente de 0 y $90^{\circ}$ ) y analizar las diferentes configuraciones faciales. Se utilizaron diversos filtros en las imágenes obteniéndose datos manejables por nuestro programa. Luego se obtuvieron los ángulos de inclinación los cuales fueron comparados con la referencia. Cuatro de las imágenes mostraron gran simetría, siendo los ángulos resultantes bastante cercanos a los valores referenciales $\left(90^{\circ}\right.$ y $\left.0^{\circ}\right)$, es decir $86.66^{\circ}$ a $89.11^{\circ}$ y $0.84^{\circ}$ a $3.39^{\circ}$, datos que alientan a futuras investigaciones.

Palabras clave: asimetría facial, eje de simetría, regresión lineal.

Evaluación de la actividad antifúngica de aceites esenciales de eucalipto, orégano, limón y mandarina frente a Cándida albicans.

Fernández, G.* Maurtua, D.*Aguilar, D. Aida, L. Delgado, C. Díaz, X. Geng, R.

*Asesores de Investigación, Proyecto GERES-UPCH

Los aceites esenciales son mezclas de diferentes compuestos químicos denominados terpenos que se encuentran en las plantas mayormente en las hojas y tallos. Diversos trabajos han mostrado el efecto antibacteriano e insecticida de los aceites, otros han estudiado los metabolitos presentes en su aceite esencial. Sin embargo, poco se ha explorado sobre su efecto antifúngico. El objetivo del presente trabajo fue evaluar el efecto fungicida frente a cepas de Cándida albicans ATCC de los aceites esenciales de eucalipto, orégano, limón y mandarina. La extracción del aceite esencial se hizo mediante destilación por arrastre con vapor de agua a la presión atmosférica y a $99^{\circ} \mathrm{C}$. Se midió la densidad del aceite mediante el método del picnómetro y el índice de refracción usando un refractómetro $\mathrm{ABBE}$ a $25^{\circ} \mathrm{C}$. Las cepas de Cándida albicans ATCC se sembraron uniformemente en placas Petri con MHA y se dejaron incubar por 24 horas. Luego se colocaron 20 microlitros del aceite esencial sobre un disco de $6 \mathrm{~mm}$ de papel filtro 3M, 20 microlitros de Clorohexedrina (patrón) y 20 microlitros de $\mathrm{H} 2 \mathrm{O}$ en otros dos separadamente. Se dejó actuar por 48 horas sobre las cepas para luego medir los halos de inhibición con una regla. La extracción del aceite esencial tuvo el siguiente rendimiento: orégano (599 g) 2 ml, eucalipto (2526 g) 10 ml, mandarina (2400 g) 2 ml, limón (2600g) 4 ml. Los diámetros promedio de los halos de inhibición fueron: orégano $4.2 \mathrm{~cm}$, eucalipto $1.3 \mathrm{~cm}$, mandarina $0.9 \mathrm{~cm}$, limón 2 $\mathrm{cm}$, clorohexedrina $1.4 \mathrm{~cm}$ y agua $0 \mathrm{~cm}$. En conclusión, el aceite esencial de orégano es el que presentó la mayor actividad antifúngica frente a la cepa Cándida albicans ATCC seguido de los aceites de eucalipto, mandarina y limón. Los componentes químicos de timol y carvacrol presentes en el aceite esencial de orégano serían los responsables de la actividad antifúngica.

Palabras clave: actividad antifúngica, Cándida albicans, aceite esencial.

Evaluación de la desmineralización del esmalte por gaseosas en dientes de primera y segunda dentición.

Fernández, G.* Borja, K. Alemán, M. Cafferata, E. Aranda, C. *Asesor de Investigación, Proyecto GERES-UPCH

La desmineralización de los dientes y la aparición de las ca- 
ries son problemas bastante frecuentes en la población infantil peruana relacionados al consumo excesivo de bebidas gaseosas y dulces, los cuales parecen ser agentes directamente vinculados debido a la presencia de ácidos y componentes que pueden producir dichos efectos. El objetivo de este trabajo fue evaluar el grado de desmineralización del esmalte de dientes de primera y segunda dentición por efecto de los ácidos contenidos en muestras de gaseosas. Se usaron 16 dientes de primera y segunda dentición y se eligieron 4 clases de gaseosas (incolora, amarilla, negra, anaranjada) catalogadas como las de mayor consumo según encuesta local. Se pesaron los dientes y se introdujeron en vasos con $25 \mathrm{ml}$ de gaseosa durante diferentes intervalos de tiempo (30 min, 1 h, 2 h y 8 h). Luego se extrajeron con una pinza, se lavaron, secaron y pesaron nuevamente. Los ensayos se hicieron por duplicado. El pH de las gaseosas fue medido usando un potenciómetro. El volumen de $25 \mathrm{ml}$ de gaseosa se valoró con solución de $\mathrm{NaOH} 0.0201 \mathrm{~N}$ antes y después de la extracción de los dientes. Dependiendo del tipo de gaseosa, se observó que para 30 minutos de exposición la pérdida de masa fue de $0.059 \%$ a $0.3 \%$, para 60 minutos fue de $0.31 \%$ a $0.55 \%$ de masa y para 8 horas la pérdida fue de $0.51 \%$ a 0.68 $\%$ de masa. Los pH obtenidos fueron 2.54 para la negra, 3.16 para la amarilla, 3.31 para la naranja y 3.38 para la blanca. Después del experimento, el contenido de ácido total (fosfórico, carbónico y otros) osciló entre 0.012 g y 0.021 g. Se concluye que las 4 gaseosas con pH ácido menor a 3.38 producen desmineralización de los dientes en mayor o menor grado, comprobado por la perdida de peso de los dientes con respecto a su peso original y se corrobora con la disminución del contenido de ácido fosfórico en las diferentes gaseosas. A la media hora de estar el diente en contacto con la gaseosa puede perder hasta un $0.3 \%$ de masa dependiendo del contenido de ácido fosfórico o cítrico de la gaseosa.

Palabras clave: desmineralización, esmalte dental, ácido fosfórico, gaseosas.

\section{Estudio comparativo de la actividad bactericida y fungicida de enjuagues bucales Listerine sobre Streptococcus mutans y Cándida albicans.}

Untiveros, G.* Maurtua, D.* Crisanto, J. Doig, M. Baca, M. Flores, N.

*Asesores de Investigación, Proyecto GERES-UPCH

La caries dental es una enfermedad que afecta a millones de personas en todas las sociedades desarrolladas y en gran número de países en desarrollo. La bacteria más importante responsable de las caries es el Streptococcus mutans que coloniza de forma persistente la superficie de los dientes y convierte el azúcar en un ácido muy agresivo que degrada la capa superior del esmalte dental. Por otra parte, la Cándida albicans, se sabe que pertenece a la flora comensal de la boca, siendo su función la de acidificar el medio oral favoreciendo la desmineralización de la matriz inorgánica de la dentina. El objetivo de esta investigación fue determinar in vitro la actividad bactericida y fungicida de los enjuagues de Listerine Menta Fresca, Listerine Whitening, Listerine Cuidado Total y Listerine Frescor Cítrico sobre la bacteria Estreptococcus Mutans ATCC 25175 y el hongo Candida Albicans ATCC 90028 . Se prepararon medios de cultivo específicos para Streptococcus mutans y para Cándida Albicans, colocándose una pequeña cantidad de estos en sus medios respectivos. Se prepararon 6 placas, cada una con seis discos en los que se agregaron 10 microlitros de Listerine Menta Fresca, Listerine Whitening, Listerine Cuidado Total y Listerine Frescor Cítrico Clorexidina y agua. Los resultados demuestran que el Listerine Whitening fue el único que tuvo la capacidad de reducir la población tanto de Streptococcus Mutans como de la Cándida Albicans.

Palabras Claves: Caries, Streptococcus Mutans, Cándida Albicans, enjuagues bucales.

\section{Reconstrucción de imágenes PET con el algoritmo OSMAPOSL usando STIR.}

Mendoza, J.* Romero, A. Villacorta, I. Romero, F. Taboada, S. *Asesor de Investigación, Proyecto GERES-UPCH

La reconstrucción de imágenes PET se realizan mediante algoritmos analítico tales como el FBP o iterativos como el OSEM el cual deriva del MLEM. El procedimiento clásico de reconstrucción de imagen es la retroproyección filtrada (FBP). Este método es rápido y sencillo siendo el método por defecto para la obtención de imágenes para diagnóstico clínico, sin embargo la resolución de la imagen se reduce por el efecto del filtrado. Los métodos iterativos de reconstrucción de imagen se han propuesto como alternativas a FBP. Estas técnicas tienen un coste computacional más alto que FBP pero producen imágenes de mejor resolución, contraste y relación señal-ruido. El objetivo de este trabajo ha sido evaluar cualitativamente la variación del número de subsets y el número de iteraciones en la resolución de imágenes PET mediante el algoritmo de reconstrucción OSMAPOSL, una variante del OSEM, implementado en el software STIR v2.0, así como en el tiempo de ejecución total del proceso de reconstrucción. Se utilizaron 2, 4 y 6 subsets y se realizaron hasta 512 subiteraciones para la reconstrucción. Las imágenes fueron visualizadas y comparadas usando el entorno AMIDE v0.9.2. El tiempo de reconstrucción fue de alrededor de 21000 segundos y se observó que la resolución mejora a medida que aumenta el número de subiteraciones. Se proyecta la evaluación cuantitativa para estudios posteriores.

Palabras clave: PET, reconstrucción de imágenes, tomografía.

Evaluación de parámetros geométricos del benchmark PET usando el entorno de simulación GATE.

Mendoza, J.* Nogueira, M. Oliveira, G. Meza, I. Neyra, M. López, L.

*Asesor de Investigación, Proyecto GERES-UPCH 
La tomografía PET permite un diagnóstico preciso por imágenes de enfermedades como leucemia, epilepsia, Parkinson, etc. además del diagnóstico temprano de procesos cancerígenos, por lo que su desarrollo tiene repercusión en aspectos económicos, sociales y tecnológicos. El punto prioritario es la obtención de imágenes con mayor resolución y menor ruido, por lo que el diseño y estudio de equipos PET mediante sistemas virtuales es una necesidad. GATE es un entorno basado en GEANT4 creado por la colaboración OpenGATE, y permite simulaciones numéricas de sistemas PET, SPECT y CT para el desarrollo de protocolos de adquisición, ensayo de algoritmos de reconstrucción y técnicas de corrección de imágenes médicas. El objetivo del trabajo fue implementar el entorno de simulación GATE v5.0 usando Scientific Linux 5.5 y variar parámetros geométricos del benchmark PET que incluye, a fin de evaluar el efecto en los valores de sensibilidad y coincidencias del sistema PET virtual. Se instalaron las fuentes de CLHEP, ROOT, MESA, GEANT4 y GATE usando una placa Intel DG41RQ, un procesador Dual Core 2.0 y 1Gb RAM, realizando la configuración necesaria. Se variaron las dimensiones X (altura) y Z (espesor) de las septas de tungteno del benchmark PET, observando el efecto en la sensibilidad y las coincidencias verdaderas, aleatorias y por dispersión. Palabras clave: GATE, PET, GEANT4, simulación.

\section{Implementación de Cluster Beowulf con Cooperative Linux} y Scientific Linux y benchmarks Skyvase y NAS.

Mendoza, J.* Mayta, J. Mogollón, G. Pérez, G. Polick, L. Quichca, K.

*Asesor de Investigación, Proyecto GERES-UPCH

La tecnología de Clúster Beowulf fue creada en la NASA a fin de solucionar problemas de cálculo intenso en corto tiempo, incrementando la velocidad de ejecución mediante la utilización en paralelo de muchos procesadores de pc's de bajo costo conectados mediante una red privada. Su uso está siendo bastante difundido en el ámbito científico, es así que el clúster más poderoso está en el Laboratorio Nacional Oak Ride - Tennessee, con 224162 procesadores AMD x86-64 Opteron Six Core 2600 MHz (10.4 GFlops), usado para el estudio de impactos del cambio climático, descomposición enzimática de la celulosa, biocombustibles, reactores de fusión, etc. Además se utilizan para modelamiento y simulación predictiva (oceanografía, pronóstico del tiempo, astrofísica, secuenciamiento del genoma), diseños de ingeniería (robots, inteligencia artificial, CAD/CAM), imágenes médicas, tomografía, entre otros. El objetivo del presente trabajo fue implementar dos clusters Beowulf, uno usando Scientific Linux 5.5 con 16 procesadores Pentium Dual Core 2.0, placas Intel DG31PR y 1Mb RAM, y otro con Cooperative Linux y Virtual Box sobre Windows XP, así como los benchmarks Skyvase y NAS usando las librerías PVMPOV v3.5, PVM v3.4.6 y openMPI v1.4.2. Se desarrollaron shell scripts para automatizar los pasos de instalación y se implementaron evaluaron los benchmarks en dos máquinas en paralelo. NAS se implementó sobre SL5.5 pero Skyvase fue probado hasta SL4.4 debido a incompatibilidades de las librerías gráficas. En ambos el tiempo de ejecución fue menor usando dos nodos frente a uno solo. Cooperative Linux fue implementado y probado con la distribución adaptada de Fedora 10.0, sin embargo no se pudo elaborar el cluster por falta de librerías de compilación ausentes en dicha distribución. Se hace necesario elaborar una distribución de Linux basada en SL5.5 compatible con Cooperative Linux y con PVMPOV3.5

Palabras clave: Clúster Beowulf, linux, benchmark, Skyvase, NAS.

\section{Pediculosis humana, IMC y factores de riesgo en el AA.HH El Trébol, Pamplona Alta, Lima.}

Cárdenas, J.* Machuca, A Jara, M Hidalgo, S Meza, A Hinostroza, S.

*Asesor de Investigación, Proyecto GERES-UPCH

La prevalencia de pediculosis y desnutrición en el área rural y urbana marginal ha sido tradicionalmente alta y las más comunes de las enfermedades pediátricas. Los objetivos del presente trabajo fueron conocer la prevalencia de pediculosis y el índice de masa corporal (IMC) en niños e identificar los factores de riesgo que promueven la prevalencia de enfermedades infecciosas en la zona de estudio. El estudio incluyó a 50 personas, entre niños y niñas con un rango etario de 3 a 14 años. Se colectó información de los índices antropométricos (talla para la edad, peso para edad y peso para la talla), se realizó un despistaje de pediculosis y se realizaron encuestas casa por casa para determinar los factores de riesgo epidemiológico. El análisis de los datos antropométricos recolectados arrojó que el 35\% posee un estado de delgadez aceptable seguido por estado normal de nutrición (27\%), el $2 \%$ presenta preobesidad y ninguno de los estudiados presenta obesidad tipo II. Respecto al índice de masa corporal para la edad, se observó un predominio del riesgo de sobrepeso (57\%) sobre la muestra estudiada. En el caso de la talla para la edad, se observó que la mayoría presenta una talla normal para la edad (57\%) sin evidencia de talla alta para la edad. Respecto del peso para la edad el $86 \%$ presenta un peso normal para la edad y no hay evidencia de sobrepeso para la edad. Se observó una prevalencia de 62\% de Pediculus humanus en la población de niños. Entre los principales factores de riesgo destacan: viviendas con piso de tierra, carencia de agua potable y desagüe, arrojo de los desechos al desmonte, malos hábitos higiénicos como jugar con tierra o no lavarse las manos antes de comer o después de ir al baño, y la alta densidad poblacional de perros y gatos como mascotas. Por otro lado, entre los factores que favorecen la desnutrición se encontró: poco conocimiento de dietas balanceadas por parte de los padres y escasos recursos económicos que hacen que algunos alimentos nutritivos no sean accesibles. Se pudo concluir que existe una alta prevalencia 
de pediculosis en la población infantil del AAHH. El Trébol de Pamplona Alta, San Juan de Miraflores, probablemente influenciada por algunos problemas nutricionales y abundantes factores de riesgo epidemiológico.

Palabras clave: Pediculosis, prevalencia, malnutrición, salud pública, pobreza.

\section{Prevalencia parasitaria en perros callejeros del AA.HH El Trébol - Pamplona Alta, Lima y su impacto en la salud públi-} ca.

Cárdenas, J.* Rojas, F. Tineo, U. Torres, A. Vilcapoma, P. Zarate, $\mathrm{T}$.

*Asesor de Investigación, Proyecto GERES-UPCH

Algunos nemátodos presentes en caninos domésticos son zoonóticos y tienen un impacto en salud pública, el cual se acrecienta por la presencia de pobres condiciones sanitarias en la población. Los objetivos del presente trabajo fueron conocer la prevalencia de parasitosis intestinal en perros callejeros, el impacto oftalmológico de la toxocariosis en niños e identificar los factores de riesgo que promueven la transmisión de infecciones parasitarias zoonóticas en la zona de estudio. Para ello, de marzo del 2010 a octubre del 2010 se realizó un estudio parasitológico en la población del AA HH El Trébol de Pamplona Alta, San Juan de Miraflores, Lima. Las muestras coprológicas $(n=80)$ fueron colectadas de la superficie arenosa de los caminos cercanos a las casas y fueron examinadas por los métodos de sedimentación de Dennis y de flotación de Faust, se empleó el examen oftalmológico para el diagnóstico de toxocariosis humana ocular y encuestas casa por casa para evaluar los factores de riesgo presentes en la zona de estudio. Se encontró que la prevalencia de Toxocara canis fue de $22.5 \%$ (29 muestras fecales positivas) y de la Toxascaris leonina fue de $2.5 \%$ (2 muestras fecales positivas). No se encontraron niños con toxocariosis ocular. Los factores de riesgo que favorecen la persistencia de toxocariosis fueron: saneamiento básico deficiente, viviendas con piso de tierra, carencia de agua potable y desagüe, y arrojo de los desechos al desmonte; y los malos hábitos higiénicos, entre los cuales destacan: jugar con la tierra y no lavarse las manos antes de comer o después de ir al baño. El contacto directo de la población infantil con los perros callejeros sugiere que aunque no se observó patología oftalmológica en los niños evaluados, la presencia de agentes etiológicos de toxocariosis y la alta concentración de factores de riesgo implican que la elevada densidad de caninos domésticos en las zonas aledañas al vecindario tienen un probable impacto en la salud pública infantil.

Palabras clave: Toxocariosis, Prevalencia, Zoonosis, Salud Publica, Caninos domésticos.

Producción de alginato con algas pardas peruanas. Languasco, J.*Agüero, T. Cano, S. Castro, K. Cuyutupac, L.
Fernández, M.

*Asesor de Investigación, Proyecto GERES-UPCH

El objetivo del presente trabajo ha sido extraer alginato de algas pardas peruanas y comprobar su estabilidad cuando es almacenado a diferentes condiciones. En Octubre del 2010 se recolectaron algas pardas phaeophyta, orden de laminariales de la especie Eisenia gracilis Dawson en la Bahía de Paracas. El procedimiento que se siguió fue el del método alcalino (Abdel Zaher 1994). Para un peso de alga seca y molida se obtuvo un peso de acido algínico de 0.51 gramos, con rendimiento del $10.2 \%$. Brito L, en Venezuela para algas pardas Sargassum vulgare, obtuvo rendimiento entre 15.4 y $15.8 \%$ usando el método del acido algínico. Acha de la Cruz en un estudio sobre la extracción de acido algínico en algas pardas phaeophyta, orden laminariales, de la especie grateolopia de la zona de Ancón obtuvo un rendimiento entre 13 y 15.8 \% dependiendo de la temperatura de extracción. Es recomendable proseguir con la investigación a fin de establecer los factores que influyen en el rendimiento del acido algínico y determinar una forma de almacenamiento para tener una mejor estabilidad dimensional del alginato de calcio.

Palabras clave: ácido algínico, alginato de calcio, algas pardas.

\section{Evaluación de la dosis en tomografía computarizada por si-} mulación Monte Carlo.

Oyardo, J.* Pizarro, T. Hashimoto, P. Quintana, S. Pérez, D. Jurado, $\mathrm{V}$.

*Asesor de Investigación, Proyecto GERES-UPCH

El método Monte Carlo es una herramienta efectiva para calcular la dosis en tomografía computarizada. La tomografía es una técnica de diagnostico que aporta dosis significativas en órganos y tejidos. El objetivo del trabajo ha sido determinar la dependencia de la dosis con el KV y con el mAs en un examen CT. Se usó el programa CTDOSE que incorpora una rutina de simulación Monte Carlo para el cálculo de la dosis efectiva en un maniquí matemático hermafrodita ADAM. Se mantuvo constante el mAs y se evaluó la respuesta de la dosis en función del KV, luego se analizó la respuesta de la dosis en función del mAs manteniendo el KV constante. Los índices de dosis CTDI fueron tomados de la bibliografía y los parámetros como espesor de corte, posición inicial y final de la exploración tomográfica fueron elegidos entre los más usados en exámenes clínicos. A partir de los resultados se obtuvo la relación lineal de la dosis con el KV y con el mAs para estas condiciones. Se concluyó que la dosis en CT es función lineal del KV y del mAs, aumentando proporcionalmente al aumentar el KV y el mAs.

Palabras Clave: Monte Carlo, rayos X, índice de dosis CTDI, dosis CT. 


\section{Simulación Monte Carlo del espectro de rayos X de una uni- dad dental.}

Oyardo, J.* Talavera, J. Scialom, P. Avilés, C. Saldaña, A. Sánchez, D.

*Asesor de Investigación, Proyecto GERES-UPCH

La radiografía dental es una técnica usada en rehabilitación oral para obtención de imágenes, siendo necesario conocer las características del haz de rayos x usado para obtener imágenes de óptima calidad. Los objetivos del presente trabajo fueron obtener mediante simulación Monte Carlo el espectro de fluencia de fotones para una unidad de rayos $\mathrm{X}$ dental Gnatus Timex 70C y evaluar el efecto de la variación del Kilovoltaje (KV) en el espectro de fluencia de fotones. Usando el programa MCNP se simuló la fuente de electrones del cátodo que inciden sobre un blanco de tungsteno con ángulo de 19o y luego se produjeron fotones que fueron dispersados atravesando la ventana de salida del tubo, el filtro de $1 \mathrm{~mm}$ de Al, el diafragma de plomo y el cilindro colimador. El espectro de fotones fue obtenido a una distancia de 20,28 cm del blanco para voltajes nominales de 70 y $100 \mathrm{KV}$ donde se pudo observar la presencia de los picos característicos del tungsteno. Se concluyó que la fluencia de fotones y la energía media aumentan cuando se aumenta el KV. El espectro calculado puede ser usado para calcular la dosis en radiología dental.

Palabras clave: Monte Carlo, espectro de fluencia de fotones, dosis. 